\title{
Effects of short-term potassium iodide treatment for thyrotoxicosis due to Graves disease in children and adolescents
}

\author{
Kyung Uk Jeong, MD, \\ Hae Sang Lee, MD, \\ Jin Soon Hwang, MD, PhD \\ Department of Pediatrics, Ajou \\ University Hospital, Ajou University \\ School of Medicine, Suwon, Korea
}

Received: 31 August, 2014

Revised: 1 October, 2014

Accepted: 11 November, 2014

\author{
Address for correspondence: \\ Jin Soon Hwang, MD, PhD \\ Department of Pediatrics, Ajou \\ University Hospital, Ajou University \\ School of Medicine, 164 World cup- \\ ro, Yeongtong-gu, Suwon 443-380, \\ Korea \\ Tel: +82-31-219-5166 \\ Fax: +82-31-219-5169 \\ E-mail: pedhwang@ajou.ac.kr
}

Purpose: Graves disease is the most common cause of hyperthyroidism in children. Inorganic iodide has been used in combination with antithyroid drugs for more effective normalization of thyroid hormones in some cases of severe thyrotoxicosis. This study aimed to investigate clinical characteristics of childhood thyrotoxicosis and effectiveness of inorganic iodide in the early phase of treatment.

Methods: Sixty-seven pediatric patients (53 girls/14 boys, $11.1 \pm 3.4$ years of age), with newly diagnosed thyrotoxicosis due to Graves disease were recruited. Fortynine patients were treated with antithyroid drugs alone, while 18 patients were treated with combination of antithyroid drugs and potassium iodide. Initial thyroid function tests and levels of thyroid antibodies were recorded for all patients. Thyroid function tests were repeated 2 and 8 weeks after the initiation of treatment. Measurement thyroid antibodies were done 8 weeks after the initiation of treatment.

Results: Mean triiodothyronine and free thyroxine levels were significantly lower $(P<0.05)$ in the group receiving combined therapy of antithyroid drugs and potassium iodide after 2 weeks of treatment compared to the patients receiving antithyroid drugs alone. Eight weeks after the initiation of treatment, thyroid function tests in the two groups did not show significant differences.

Conclusion: The use of potassium iodide in combination with antithyroid drug is effective for more rapid normalization of thyroid hormones in the early phase treatment of childhood thyrotoxicosis, but larger studies with adequate power are needed in future.

Keywords: Thyrotoxicosis, Graves disease, Potassium iodide, Child

\section{Introduction}

Thyrotoxicosis is a disorder of excessive thyroid hormone production, while hyperthyroidism specifically refers to the increased synthesis and secretion of thyroid hormone. The most common cause of thyrotoxicosis is Graves disease (GD), in which autoantibodies bind to and stimulate thyroid-stimulating hormone (TSH) receptors on the surface of thyroid follicular cells, resulting in excessive production and release of triiodothyronine (T3) and thyroxine (T4) ${ }^{1)}$. GD is the most common cause of hyperthyroidism in children ${ }^{2)}$, accounting for approximately $10 \%-15 \%$ of childhood thyroid diseases ${ }^{3)}$. The incidence of GD rises from 0.1 per 100,000 in young children to 3 per 100,000 in adolescence ${ }^{4)}$ with higher annual global rates of 14.1 per 100,000 including Hong Kong ${ }^{5}$.

Although mortality is rare, thyrotoxicosis is associated with palpitations, weight loss, impaired skeletal mineralization, behavioral problems, poor academic performance, and detrimental effects on children and adolescents ${ }^{3,6)}$. Prompt and aggressive treatment 
of hyperthyroidism is essential to avoid these associated mortality and morbidity. Antithyroid drugs (ATD), surgical resection, and radioactive iodine therapy are three major treatment options for Graves hyperthyroidism. Although most pediatric endocrinologists agree that ATDs should be the first line of treatment in children, the optimal treatment for hyperthyroidism remains controversial ${ }^{7)}$.

Inorganic iodide has been used in combination with ATDs to achieve rapid normalization of thyroid hormone levels in severe thyrotoxicosis or as a pretreatment before urgent thyroidectomy ${ }^{8)}$. While previous studies have not shown treatment effectiveness using combined ATDs and potassium iodide (KI) to be superior to ATDs alone ${ }^{9,10)}$, some recent studies reported improved short-term control of Graves hyperthyroidism in adults with combined methimazole (MMI) and KI treatment ${ }^{11)}$. The purpose of the present study was to investigate clinical characteristics of childhood thyrotoxicosis and effectiveness of KI as an early phase treatment.

\section{Materials and methods}

\section{Subjects}

Sixty-seven pediatric patients with newly diagnosed thyrotoxicosis due to GD were recruited at Department of Pediatrics, Ajou University Hospital from January 2003 to December 2012. GD was diagnosed based on clinical symptoms and confirmed using elevated levels of serum free T4 and T3, suppressed TSH levels, and positive TSH receptor-stimulating antibodies. Patients with toxic adenoma, multinodular goiter or autoimmune thyroiditis were excluded.

\section{Methods}

January 2003 to December 2012 charts belonging to patients under 17 years of age $(n=67)$ were retrospectively reviewed. All patients were initially given ATDs, propylthiouracil (PTU), at a dose of $5-10 \mathrm{mg} / \mathrm{kg} /$ day in 55 patients (82\%) and MMI, at a dose of $0.3-0.8 \mathrm{mg} / \mathrm{kg} /$ day in 12 patients (18\%). Among the 67 patients, 49 patients (73\%), who were diagnosed as GD between January 2003 and June 2009, were treated with ATD alone while 18 patients (27\%), who were diagnosed between July 2009 and December 2012, were treated with combination of ATD and KI. The categorization of the two groups was performed on the basis of the period, since the policy of combination treatment of ATD and $\mathrm{KI}$ for childhood thyrotoxicosis was implemented from July 2009 in our center. Lugol's solution, a saturated solution of $\mathrm{KI}$, was administered three times daily, $0.25 \mathrm{~mL}$ ( $25 \mathrm{mg}$ of $\mathrm{KI}$ ) at a time, in combination with ATD during the first three days of treatment. There were no differences between the groups with respect to the doses of ATDs administered.

Initial levels of serum free T4, T3, TSH, and thyroid antibodies (antimicrosomal antibody, antithyroglobulin antibody and TSH receptor-stimulating antibody) were recorded for all included patients. To evaluate the effectiveness of initial treatment, thyroid function tests (TFTs) were repeated two weeks after the initiation of ATDs while TFTs and measurement of thyroid antibodies were done eight weeks later. The reference ranges for free T4, T3, and TSH were $0.64-1.72 \mathrm{ng} / \mathrm{dL}, 76-190 \mathrm{ng} / \mathrm{dL}$, and $0.15-5.00 \mu \mathrm{IU} / \mathrm{mL}$, respectively. The reference ranges for antimicrosomal antibody, antithyroglobulin antibody, and TSH receptor-stimulating antibody were $0-60 \mathrm{U} / \mathrm{ml}, 0-60 \mathrm{U} / \mathrm{mL}$, and 0-1.5 IU/L. Serum free T4, T3, and TSH concentrations were measured by radioimmunoassay methods (Auto RIA/SR300, Startec Biomedical AG, Birkenfeld, Germany). Antimicrosomal antibody and antithyroglobulin antibody were measured by immunoradiometric assays (Packard Cobra II Gamma Counter, Perkin Elmer Life Sciences, Courtaboeuf, France).

Information was obtained regarding age at identification, gender, clinical symptoms and signs, family history of thyroid diseases, heights, weights and body mass index (BMI).

\section{Statistics}

The levels of serum free T4, T3, TSH and thyroid antibodies among the patients treated with ATD alone and those

Table 1. Characteristics in 67 children and adolescents at onset of thyrotoxicosis

\begin{tabular}{|c|c|c|c|c|}
\hline Characteristic & Total $(n=67)$ & $\operatorname{ATD}(n=49)$ & ATD+KI $(n=18)$ & $P$-value \\
\hline Girls & 53 & 39 & 14 & \\
\hline Boys & 14 & 10 & 4 & \\
\hline Girls:boys & $3.7: 1$ & $3.9: 1$ & $3.5: 1$ & \\
\hline Age at diagnosis (yr) & $11.1 \pm 3.4$ & $11.1 \pm 3.5$ & $11.2 \pm 3.2$ & 0.944 \\
\hline Family history of thyroid diseases (\%) & 47.7 & 48.9 & 44.4 & 0.690 \\
\hline Weight (kg) & $40.2 \pm 13.9$ & $39.9 \pm 14.1$ & $41.2 \pm 13.9$ & 0.736 \\
\hline Height (cm) & $146.9 \pm 21.6$ & $146.4 \pm 22.3$ & $148.6 \pm 20.2$ & 0.707 \\
\hline Body mass index $\left(\mathrm{kg} / \mathrm{m}^{2}\right)$ & $17.7 \pm 2.6$ & $17.7 \pm 2.6$ & $17.9 \pm 2.7$ & 0.783 \\
\hline \multicolumn{5}{|l|}{ Initial thyroid function test } \\
\hline Free T4 (ng/dL) & $3.89 \pm 1.27$ & $3.74 \pm 1.21$ & $4.32 \pm 1.35$ & 0.101 \\
\hline $\mathrm{T} 3$ (ng/dL) & $439 \pm 247$ & $415 \pm 195$ & $503 \pm 351$ & 0.200 \\
\hline TSH $(\mu \mathrm{IU} / \mathrm{mL})$ & $0.042 \pm 0.043$ & $0.038 \pm 0.042$ & $0.051 \pm 0.045$ & 0.310 \\
\hline
\end{tabular}

Values are presented as mean \pm standard deviation unless otherwise indicated.

ATD, antithyroid drug; Kl, potassium iodide; T4, thyroxine; T3, triiodothyronine; TSH, thyroid-stimulating hormone. 
treated with combination of ATD and Lugol's solution, were compared using independent $T$-test. The data were expressed as mean \pm standard deviation and a $P$-value of less than 0.05 was considered significant.

\section{Results}

Of the 67 children, 53 were girls (79\%) while 14 (21\%) were boys. The average age at onset was $11.1 \pm 3.4$ years. A positive family history was obtained in 32 patients (47.7\%). A first-degree relative was involved in 20 patients (30\%): 4 simple goiter, 11 hyperthyroidism, 3 hypothyroidism, 1 thyroiditis, and 1 thyroid cancer. The mean body weight and height were $40.2 \pm 13.9 \mathrm{~kg}$ and $146.9 \pm 21.6 \mathrm{~cm}$, respectively (Table 1).

Sixty children $(89.6 \%)$ presented with goiter while ophthalmopathy was found in 34 children (50.7\%) (Table 2). All patients had increased serum free T4 (mean, $3.89 \mathrm{ng} / \mathrm{dL}$; range, $1.23-7.98 \mathrm{ng} / \mathrm{dL}$ ), T3 (mean, $439 \mathrm{ng} / \mathrm{dL}$; range $108-1748 \mathrm{ng} / \mathrm{dL}$ ) and suppressed TSH levels (mean, $0.04 \mu \mathrm{IU} / \mathrm{mL}$; range, 0.01-0.19 $\mu \mathrm{IU} / \mathrm{mL})$ at initial diagnosis.

All patients $(\mathrm{n}=67)$ received ATDs (either PTU or MMI) while 18 patients $(27 \%)$ were treated with a combination of ATD and KI. The levels of thyroid antibodies at initial presentation

Table 2. Presenting features of children and adolescents with thyrotoxicosis

\begin{tabular}{lc}
\hline Symptoms and signs & No. of patients (\%) \\
\hline Goiter & $60(89.6)$ \\
Exophthalmos & $34(50.7)$ \\
Weight loss & $30(44.8)$ \\
Tachycardia & $30(44.8)$ \\
Palpitation & $28(41.8)$ \\
Sweating & $25(37.3)$ \\
Hypertension & $24(35.8)$ \\
Fatigue & $22(32.8)$ \\
Heat intolerance & $19(28.4)$ \\
Diarrhea & $16(23.9)$ \\
Fever & $5(7.5)$ \\
Dyspnea & $3(4.5)$ \\
\hline
\end{tabular}

and eight weeks later demonstrated no significant differences between groups (Table 3). No significant differences were found between serum levels of free T4, T3, and TSH at initial diagnosis, but mean free T4 values were significantly lower $(P<0.05)$ in the group receiving combined therapy of $\mathrm{ATD}+\mathrm{KI}$, after two weeks of treatment (Fig. 1A). Similarly, serum T3 levels rapidly decreased after two weeks of treatment and mean levels were significantly lower $(P<0.05)$ in the group receiving combined therapy compared with those of the patients receiving ATD alone (Fig. 1B). Serum TSH levels after two weeks of treatment showed no significant differences between two groups (Fig. 1C). Serum levels of free T4, T3, and TSH showed no significant differences between two groups after eight weeks of treatment. No treatment-related adverse effects such as hypothyroidism were observed in both groups.

\section{Discussion}

The current study assessed clinical characteristics of 67 pediatric patients diagnosed with thyrotoxicosis due to GD. Additionally, ATD monotherapy was compared with combined treatment of ATD and KI in terms of rapid normalization of thyroid hormones during the early phase. Most patients were female with an average age at presentation of 11.1 years,

Table 3. Serum levels of thyroid antibodies at onset and after 8 weeks of treatment

\begin{tabular}{lccc}
\hline & ATD alone & ATD $+\mathrm{Kl}$ & P-value \\
\hline At onset & & & \\
Antimicrosomal Ab (U/mL) & $2,280 \pm 2,117$ & $1,964 \pm 1,769$ & 0.575 \\
Antithyroglobulin $\mathrm{Ab}(\mathrm{U} / \mathrm{mL})$ & $683 \pm 1600$ & $627 \pm 925$ & 0.889 \\
TSH receptor-stimulating Ab (IU/L) & $70 \pm 85$ & $80 \pm 94$ & 0.677 \\
After 8 weeks of treatment & & & \\
Antimicrosomal Ab (U/mL) & $2,355 \pm 2,130$ & $1,540 \pm 1,651$ & 0.150 \\
Antithyroglobulin $\mathrm{Ab}(\mathrm{U} / \mathrm{mL})$ & $590 \pm 998$ & $566 \pm 978$ & 0.930 \\
TSH receptor-stimulating Ab (IU/L) & $59 \pm 78$ & $46 \pm 42$ & 0.510 \\
\hline
\end{tabular}

Values are presented as mean \pm standard deviation.

ATD, antithyroid drug; KI, potassium iodide; $\mathrm{Ab}$, antibody; TSH, thyroid-stimulating hormone.
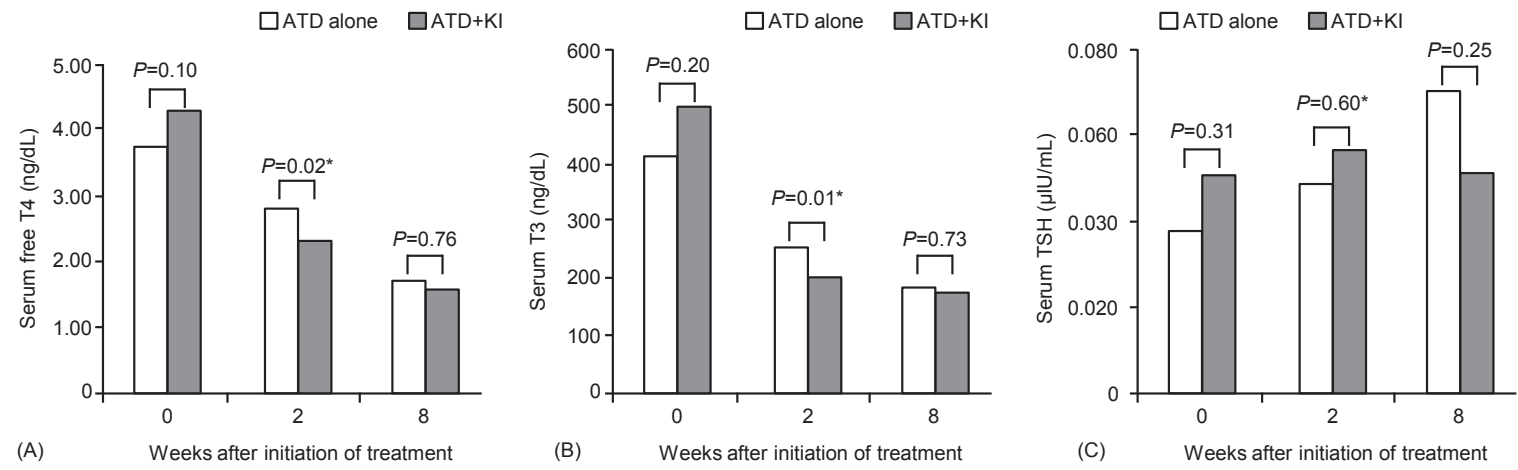

Fig. 1. Serial changes in serum free T4 (A), T3 (B), and TSH (C) in two groups. Open column indicates ATD alone group and shaded column indicates ATD $+K$ K group. Significantly different at $P<0.05\left(^{*}\right)$ between groups receiving ATD alone and those receiving combined therapy. T3, triiodothyronine; T4, thyroxine; TSH, thyroid-stimulating hormone; ATD, antithyroid drug; Kl, potassium iodide. 
which was comparable to other studies ${ }^{12}$. The common clinical features observed in the patients at initial diagnosis were goiter (89.6\%), exophthalmos (50.7\%), weight loss (44.8\%), tachycardia (44.8\%), and palpitation (41.8\%), which were similar to those noted in adults ${ }^{1)}$. Although assessments of personality change or abnormal behavior were not included in our study, previous studies have reported behavioral problems such as lack of concentration, delayed advancement in school, perversity and enuresis $^{13)}$, as presenting features of thyrotoxicosis in children. When combined ATD and KI treatment was compared with ATD monotherapy, we clearly observed more effective reduction of free T4 and T3 by combined therapy than ATD monotherapy after 2 weeks of treatment. However, there was no significant difference in the serum free $\mathrm{T} 4$ and $\mathrm{T} 3$ values between two groups after 8 weeks of treatment. A previous study described that the beneficial effect of KI in hyperthyroidism was only temporary ${ }^{14}$. Moreover, another study reported that KI treatment alone in GD produced only short term control of severe symptoms in hyperthyroidism and that its use hindered the efficacy of following treatment with $\mathrm{ATD}^{15}$. In a recent Italian study, the combination of ATD with KI was not superior to ATD alone in the normalization of serum thyroid hormone concentrations. On the other hand, serum T3 levels as well as heart rates were more rapidly reduced when sodium ipodate was added to ATD ${ }^{10}$. A more recent study showed that combined treatment with ATD and KI enhanced the short-term control of Graves hyperthyroidism in adults ${ }^{11)}$, but there have been few reports describing the effect of combination therapy of ATD with KI in childhood thyrotoxicosis.

A decreased secretion of thyroid hormones by inorganic iodine could have been brought about in several ways including an inhibition of thyroid hormone synthesis, a decrease in the fractional rate of hormone release, or both. The inhibition of organic binding of iodide in the thyroid gland by administration of a large amount of nonradioactive iodide, resulting in the reduction of thyroid hormone synthesis, is termed as the WolffChaikoff effect (WCE). This effect can occur in patients with thyrotoxicosis ${ }^{16)}$. Inorganic iodide is absorbed rapidly into the circulation and by the thyroid. In larger amounts, it immediately blocks further uptake of iodide by several means, particularly by saturating the iodide transport mechanisms of the thyroid, and by inhibiting the intrathyroidal organification of iodide. Although the exact mechanism of WCE remains speculative, elevated intrathyroidal iodide concentrations apparently induce decreased availability of hydrogen peroxide for iodination reactions ${ }^{177}$. Even if a decrease in synthesis occurs during iodide administration, several lines of evidence indicate that the major effect of iodide is to inhibit the release of thyroid hormones ${ }^{18)}$. At the present time, iodide is primarily used in conjugation with ATDs to prepare patients for thyroidectomy ${ }^{19)}$, rather than the use of iodide alone as definitive therapy for thyrotoxicosis.

In this study, more rapid reduction of thyroid hormones by combination therapy with ATDs and KI than with ATD monotherapy was observed in children and adolescents diagnosed as thyrotoxicosis due to GD. Although it is well known that use of $\mathrm{KI}$ as monotherapy may result in escape from control of thyrotoxicosis ${ }^{20)}$, our study shows that this does not occur when KI is administered simultaneously with ATDs. We did not observe a reduction in the efficacy of ATDs by KI.

The study limitations include the retrospective use of clinical data, the relatively small number of patients and the use data at one institution. However, this is the first report on the effect of combination treatment of inorganic iodide and ATD in childhood thyrotoxicosis, and we expect that double-blinded randomized controlled studies would provide more compelling outcome in future.

In conclusion, more rapid normalization of thyroid hormones was achieved in the group with combined treatment of ATD and Lugol's solution, than the group treated with ATD alone in our 10-year retrospective study. Although no statistical difference was found between the two groups in later followup evaluations, we suggest that the use of Lugol's solution in combination with ATD during the initial phase of treatment is beneficial in alleviating the symptoms and signs by more effective normalization of thyroid hormones in childhood thyrotoxicosis. A larger study with adequate power should be undertaken to examine the effect of Lugol's solution in childhood thyrotoxicosis.

\section{Conflict of interest}

No potential conflict of interest relevant to this article was reported.

\section{References}

1. Franklyn JA, Boelaert K. Thyrotoxicosis. Lancet 2012;379:1155-66.

2. Rivkees SA. Pediatric Graves' disease: controversies in management. Horm Res Paediatr 2010;74:305-11.

3. Zimmerman D, Lteif AN. Thyrotoxicosis in children. Endocrinol Metab Clin North Am 1998;27:109-26.

4. Lavard L, Ranlov I, Perrild H, Andersen O, Jacobsen BB. Incidence of juvenile thyrotoxicosis in Denmark, 19821988. A nationwide study. Eur J Endocrinol 1994;130:5658.

5. Wong GW, Cheng PS. Increasing incidence of childhood Graves' disease in Hong Kong: a follow-up study. Clin Endocrinol (Oxf) 2001;54:547-50.

6. Fisher DA. Graves' disease in children. Curr Ther Endocrinol Metab 1994;5:71-4.

7. Vanderpump MP, Ahlquist JA, Franklyn JA, Clayton RN. Consensus statement for good practice and audit measures in the management of hypothyroidism and hyperthyroidism. The Research Unit of the Royal College of Physicians of London, the Endocrinology and Diabetes Committee of the Royal College of Physicians of London, and the Society for Endocrinology. BMJ 1996;313:539-44.

8. Nayak B, Burman K. Thyrotoxicosis and thyroid storm. Endocrinol Metab Clin North Am 2006;35:663-86. 
9. Croxson MS, Hall TD, Nicoloff JT. Combination drug therapy for treatment of hyperthyroid Grave's disease. J Clin Endocrinol Metab 1977;45:623-30.

10. Roti E, Robuschi G, Gardini E, Montermini M, Salvi M, Manfredi A, et al. Comparison of methimazole, methimazole and sodium ipodate, and methimazole and saturated solution of potassium iodide in the early treatment of hyperthyroid Graves' disease. Clin Endocrinol (Oxf) 1988; 28:305-14.

11. Takata K, Amino N, Kubota S, Sasaki I, Nishihara E, Kudo $\mathrm{T}$, et al. Benefit of short-term iodide supplementation to antithyroid drug treatment of thyrotoxicosis due to Graves' disease. Clin Endocrinol (Oxf) 2010;72:845-50.

12. Gruneiro-Papendieck L, Chiesa A, Finkielstain G, Heinrich JJ. Pediatric Graves' disease: outcome and treatment. J Pediatr Endocrinol Metab 2003;16:1249-55.

13. Mckendrick T, Newns GH. Thyrotoxicosis in children: a follow-up study. Arch Dis Child 1965;40:71-6.

14. Frazier $\mathrm{CH}$. The use of potassium iodide in hyperthyroidism. Ann Surg 1932;95:517-24.
15. Martino E, Balzano S, Bartalena L, Loviselli A, Sica V, Petrini $\mathrm{L}$, et al. Therapy of Graves' disease with sodium ipodate is associated with a high recurrence rate of hyperthyroidism. J Endocrinol Invest 1991;14:847-51.

16. Harden RM, Koutras DA, Alexander WD, Wayne EJ. Quantitative studies of iodine metabolism in iodide-treated thyrotoxicosis. Clin Sci 1964;27:399-405.

17. Chiraseveenuprapund P, Rosenberg IN. Effects of hydrogen peroxide-generating systems on the Wolff-Chaikoff effect. Endocrinology 1981;109:2095-102.

18. Wartofsky L, Ransil BJ, Ingbar SH. Inhibition by iodine of the release of thyroxine from the thyroid glands of patients with thyrotoxicosis. J Clin Invest 1970;49:78-86.

19. Feek CM, Sawers JS, Irvine WJ, Beckett GJ, Ratcliffe WA, Toft AD. Combination of potassium iodide and propranolol in preparation of patients with Graves' disease for thyroid surgery. N Engl J Med 1980;302:883-5.

20. Woeber KA. Iodine and thyroid disease. Med Clin North Am 1991;75:169-78. 